\title{
Growth and characterization of large, high quality cubic diamond crystals
}

\author{
ZANG ChuanYi $^{1 *}$, LI Ming $^{1} \&$ CHEN LunJian ${ }^{2}$ \\ ${ }^{1}$ School of Physics and Chemistry, Henan Polytechnic University, Jiaozuo 454000, China; \\ ${ }^{2}$ Institute of Materials Science and Engineering, Henan Polytechnic University, Jiaozuo 454000, China
}

Received September 5, 2011; accepted October 27, 2011; published online March 8, 2012

\begin{abstract}
High quality cubic diamond crystals were grown using the temperature gradient method at high pressure and high temperature (HPHT), in a new FeNi alloy as solvent. The crystals were grown at relatively low temperatures suitable for the growth of $\{100\}$ faces. An increase in the radial growth rate, and inhibition of the axial growth caused the growth of large, high quality cubic diamond single crystals at high growth rates. For example, over $33 \mathrm{~h}$, the radial growth rate was $0.22 \mathrm{~mm} / \mathrm{h}$, while the axial growth rate was only $0.08 \mathrm{~mm} / \mathrm{h}$; the growth rate by weight was also increased to $7.3 \mathrm{mg} / \mathrm{h}$. The yellow color of our crystal samples was more uniform than samples from Sumitomo Corporation of Japan and Element Six Corp. The Raman FWHW of the $1332 \mathrm{~cm}^{-1}$ peak in our diamond sample was smaller than the Element Six Corp. sample, but larger than that of the Sumitomo Corp. sample. The nitrogen content of our diamond samples was $240 \mathrm{ppm}$, which was much higher than the Sumitomo and Element Six samples because of the higher growth rate of our diamond samples.
\end{abstract}

HPHT, diamond, temperature gradient method, growth rate

Citation: Zang C Y, Li M, Chen L J. Growth and characterization of large, high quality cubic diamond crystals. Chin Sci Bull, 2012, 57: 1733-1738, doi: $10.1007 / \mathrm{s} 11434-012-5023-4$

Diamond is a covalent crystal in which carbon atoms are strongly bonded to each other. This single structural feature leads to the highest hardness among all natural materials and gives it superior thermal conductivity, as well as excellent chemical stability and transparency. Diamond has been employed in a wide range of uses that exploit these properties. Large diamond crystals on the order of several millimeters across are widely used for high-precision and superprecision cutting tools for precision cutting nonferrous metals, such as aluminum and copper, and soft materials, like plastics. For cutting tools, thin plates of diamond are ideal for attachment to tools by brazing. The $\{100\}$ cubic plane orientation of the blanks offers easy grinding during tool edge preparation, and the orientation defined edge is ideal for most cutting tool applications. Therefore, most diamond crystals in the current market are provided with this cubic morphology; almost no other faces, such as $\{111\},\{110\}$ can

*Corresponding author (email: dndzcy@hpu.edu.cn; dndzcy@ sina.com) be found. On the other hand, $\{111\}$ and $\{110\}$ faces are common, even dominant in natural diamonds, while synthetic diamond crystals characteristically display $\{111\}$ and $\{100\}$ faces [1,2] and often show a cube-octahedral morphology. Thus the only way to obtain cubic diamond crystals with well-developed $\{100\}$ faces dominant, is by synthesis. Large, high-quality single crystal diamond is mostly grown under high pressure and high temperature (HPHT) using the temperature gradient method developed by GE Corp. in 1970 [3-8]. The CVD method has recently found favor for the growth of large synthetic diamond crystals and has been developing rapidly. One of the most important aspects of this technique is that high-quality diamond single crystals must be used as the growth substrate. These "seeds" must be composed of only $\{100\}$ faces, and are currently obtained by HPHT synthesis. In general, the larger the substrate is, the higher the growth rate of crystals grown by CVD. So it could be said that the development of CVD is dependent on HPHT methods $[9,10]$. 
The growth of cubic crystals is considered more difficult than cube-octahedral diamond crystals. First, the growth temperatures that ensure the growth of high-quality cubic diamond crystals have a smaller interval when growing $\{100\}$ faces, and this has been reported to be only $10 \mathrm{~K}$ $[11,12]$. Second, in the temperature gradient method the crystal starts its growth in the low temperature region, as the crystal grows larger, the crystal extends into the higher temperature region. This can change the morphology of the crystal from cube into cube-octahedron.

In this study, using the temperature gradient method under HPHT, in a FeNi alloy as metal solvent, high-quality cubic diamond crystals with a $\{100\}$ growth face have been grown, at growth rates up to $7.3 \mathrm{mg} / \mathrm{h}$. This is higher than the best growth rate for the same growth period reported by Sumitomo Corporation of Japan (Sumitomo Corp. for short). The performance of the synthetic diamond crystals from our lab have also been analyzed and compared with other diamond samples.

All diamond crystals were grown using the temperature gradient method under HPHT at $5.4 \mathrm{GPa}$ and $1500 \mathrm{~K}$ produced in a China made cubic anvil high pressure apparatus. The schematic diagram is shown in Figure 1. High purity graphite powder $(99.99 \%)$ was used as the carbon source and was placed in the higher temperature region of the growth cell. High quality synthetic type $\mathrm{Ib}$ diamond grits about $0.8 \mathrm{~mm}$ across were used as seed crystals, with a $\{100\}$ surface about $0.5 \mathrm{~mm} \times 0.5 \mathrm{~mm}$ as the growth surface, were placed in the lower temperature region of the growth cell. The conventional $\mathrm{Fe}_{70} \mathrm{Ni}_{30}$ alloy and a new in-house $\mathrm{FeNi}$ alloy were used as the solvent metal respectively, which were placed between the carbon source and seed crystals. After HPHT synthesis, the temperature and pressure were reduced and the samples were treated with hot dilute nitric acid to separate the diamond crystals from the FeNi solvent. The crystals were then boiled in concentrated sulfuric and nitric acid mixture for $3 \mathrm{~h}$, to remove any residual graphite and solvent on the surfaces of the crystals.

To characterize and compare our cubic diamond samples with other samples, high quality cubic diamond crystals were purchased from Sumitomo Corporation of Japan and Element Six Corp. of the UK. The appearance and color of all diamond crystals were compared with a OLYMPUS SZ61 optical microscope. Raman spectra were measured using a Renishaw laser Raman spectrometer. Micro-infrared characterization was carried out on a Bruker Optics/IFS66V infrared spectrometer and Hyperion 3000 microscope, with a laser spot size no more than $10 \mu \mathrm{m}$.

As the morphology of a grown crystal is related to the temperature at which it was grown by HPHT synthesis, the crystals can show changes in morphology from cubic to cube-octahedron to octahedron as the growth temperature increases. That is to say, $\{100\}$ faces develop more at lower temperatures [13]. However, the temperature interval at which the desired form is dominant, is very narrow, about

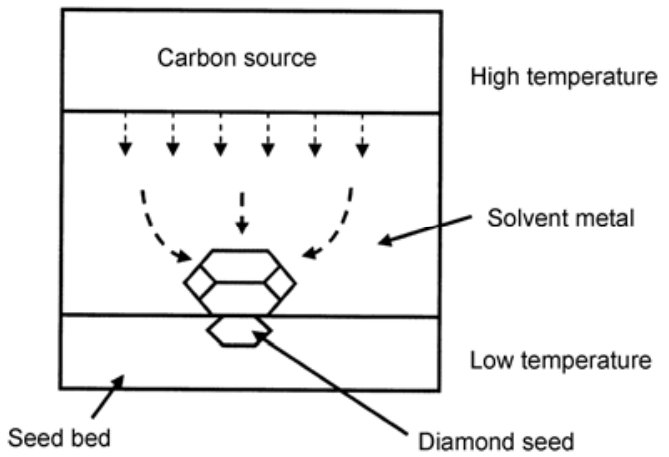

Figure 1 Assembly schematic diagram for the temperature gradient method.

$10 \mathrm{~K}$, and this means that a very precise temperature control technique is required. For prolonged growth periods this is very difficult because of phase transformations of the pressure mediums, minor changes of the external environment and fluctuations in the growth temperatures inside the growth cell. Any such change leads to a degradation in the quality of the cubic crystals. Higher temperatures cause the crystals to show a cube-octahedral morphology, and if the growth rate is high, metal inclusions and other impurities can be incorporated into crystals. At lower temperatures in the growth cell, black or skeleton crystals are obtained instead of high quality cubic diamond crystals. Just such a crystal was obtained when a conventional Fe-Ni alloy solvent was used in our setup at $5.4 \mathrm{GPa}$ and $1500 \mathrm{~K}$ for $15 \mathrm{~h}$, as shown in Figure 2.

To grow high quality cubic diamond crystals at low temperatures, the most effective strategy is to enlarge the temperature interval suitable for growing cubic diamond crystals with the $\{100\}$ faces dominant, and to use a assembly with high temperature stability for the HPHT growth. The pressures available in the China made cubic anvil high pressure apparatus are unfortunately limited, as the diamond growth temperature interval increases with increasing pressure,. The temperature interval is thankfully also related to the metal solvent, so a new FeNi alloy solvent was developed, with some trace elements as additives to improve the

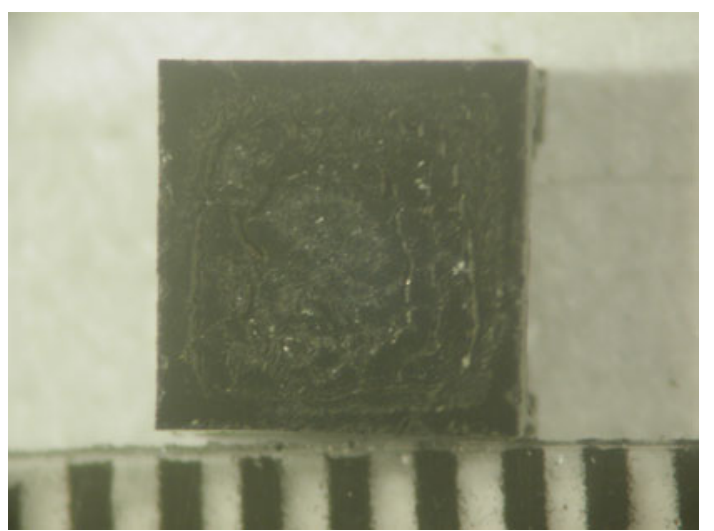

Figure 2 (Color online) Black skeleton crystal grown at a lower temperature (the smallest graduation of the scale is $1.0 \mathrm{~mm}$ ). 
catalytic performance. Compared with a conventional FeNi alloy solvent, this new metal solvent enlarges the temperature interval to $20 \mathrm{~K}$. With this new solvent, high quality cubic diamond crystals were grown at $5.4 \mathrm{GPa}$ and $1500 \mathrm{~K}$ over a number of different time periods.

All single crystals showed complete cubic shapes with the $\{100\}$ face dominant, as shown in Figure 3, and the specific growth characteristics are listed in Table 1. From the point of quality, all crystals were clear and a transparent yellow color, with smooth growth surfaces. No inclusions were observed under a microscope.

At a high growth rate, many large inclusions tend to be trapped into crystals, especially in the region directly above the seed. These seed related inclusions can be effectively avoided by keeping the relative growth rate in the vertical direction lower than in the horizontal direction. The growth rates at the surface can be controlled by adjusting the growth temperature or crystal morphology. For cubic crystals, the radial growth rate (horizontal direction) is significantly larger than the axial growth rate (vertical direction), and the radial growth rates will reduce as the growth pro- gresses. For example, at $20 \mathrm{~h}$, the radial rate is $0.30 \mathrm{~mm} / \mathrm{h}$, but at $33 \mathrm{~h}$, the radial rate has reduced to $0.22 \mathrm{~mm} / \mathrm{h}$. In comparison the axial growth rate is constant at only 0.08 $\mathrm{mm} / \mathrm{h}$, as shown in Table 1 . Therefore, because of the great difference between radial and axial growth rates, the crystal is $7.3 \mathrm{~mm}$ across, but weighs only $1.2 \mathrm{ct}$.

Focusing on the weight increase, over $33 \mathrm{~h}$ starting from $0.8 \mathrm{~mm}$ seed crystals with $\{100\}$ growth surfaces about $0.5 \mathrm{~mm} \times 0.5 \mathrm{~mm}$, the growth rate reached $7.3 \mathrm{mg} / \mathrm{h}$ (Table 1). In terms of growth techniques for large synthetic diamond crystals under HPHT, Sumitomo Corporation of Japan generally represents the highest level of technology $[11,12]$. However, as shown in Figure 6, using $0.5 \mathrm{~mm} \times 0.5 \mathrm{~mm}$ seeds and using a Fe-Co alloy as metal solvent over $30 \mathrm{~h}$, their best growth rate is only $2.0 \mathrm{mg} / \mathrm{h}$. In 2000, on large $5.0 \mathrm{~mm} \times 5.0 \mathrm{~mm}$ seed crystals, Sumiya et al. [10] have grown high quality diamond single crystals as large as $10 \mathrm{ct}$, at growth rates up to $12 \mathrm{mg} / \mathrm{h}$. However, when averaged over $30 \mathrm{~h}$, this becomes about $6.7 \mathrm{mg} / \mathrm{h}$. Thus, over the same time, the growth rate of high quality cubic diamond crystals in our lab is higher than that of Sumitomo Corporation, even

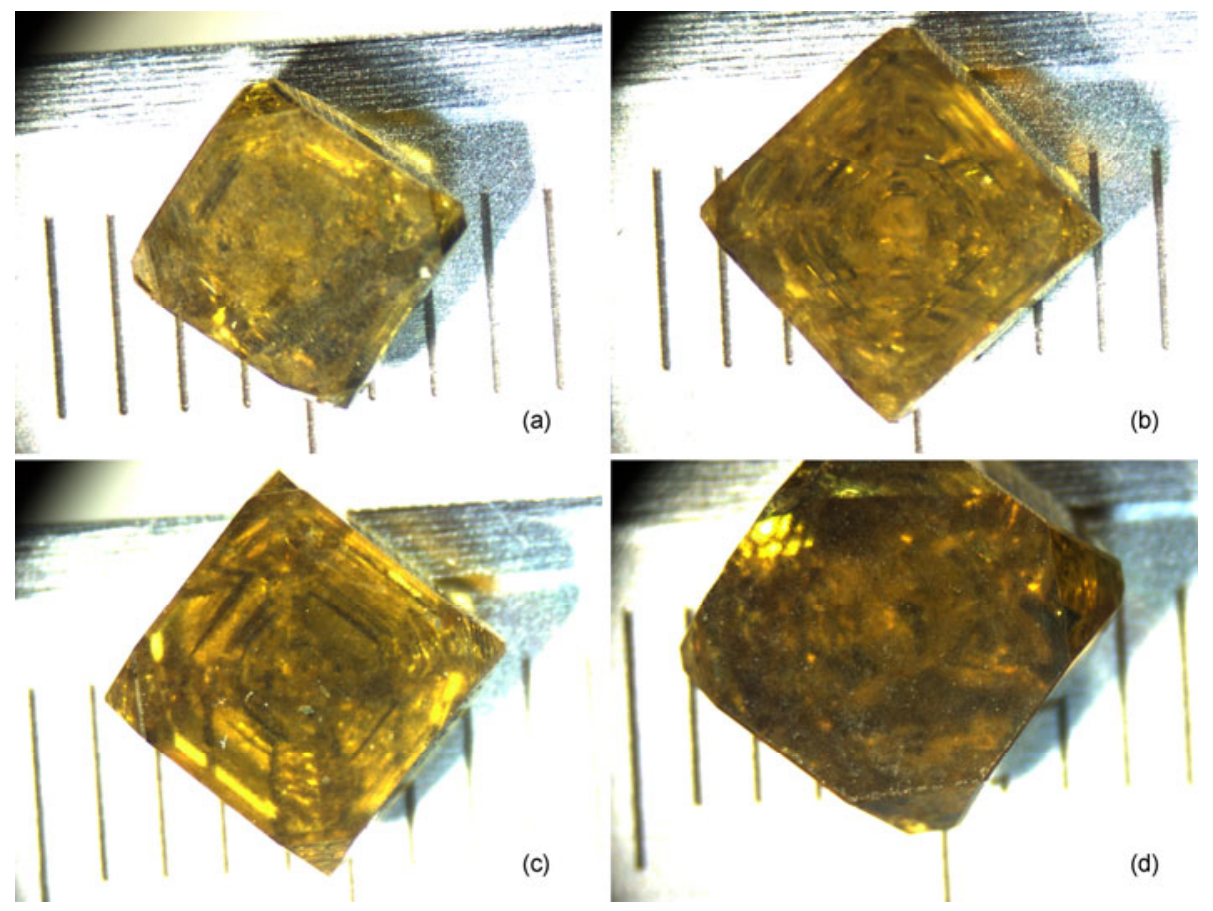

Figure 3 (Color online) High-quality cubic large diamond crystals grown over different times (the smallest graduation of the scale is $1.0 \mathrm{~mm}$ ). (a) $15 \mathrm{~h}$; (b) $20 \mathrm{~h}$; (c) $22 \mathrm{~h}$; (d) $33 \mathrm{~h}$.

Table 1 Crystal characters and growth rates of large cubic diamond crystals grown over different times

\begin{tabular}{|c|c|c|c|c|c|c|}
\hline Growth time (h) & Crystal size (mm) & Crystal weight (ct) & Radial rate $(\mathrm{mm} / \mathrm{h})$ & Axial rate $(\mathrm{mm} / \mathrm{h})$ & Growth rate $(\mathrm{mg} / \mathrm{h})$ & Inclusion \\
\hline 15 & 5.0 & 0.4 & 0.33 & 0.03 & 5.3 & No \\
\hline 20 & 6.0 & 0.7 & 0.30 & 0.08 & 7.0 & No \\
\hline 22 & 6.2 & 0.8 & 0.28 & 0.08 & 7.2 & No \\
\hline 33 & 7.3 & 1.2 & 0.22 & 0.08 & 7.3 & No \\
\hline
\end{tabular}


when compared with large-seed-crystals. Large seed crystals also suffer from the more facile inclusion of metal and other impurities into the crystals.

Synthetic diamonds tend to take up nitrogen at different concentrations for each type of growth sector. A growth sector is a three dimensional region of the crystal with a common crystallographic growth plane. The color of the diamond crystal is directly related to the nitrogen concentrations. Thus, color uniformity is one of the major characteristics to evaluate the performance of high quality cubic diamond crystals.

To distinguish minor differences in the color uniformity, after fine grinding, the rough growth surfaces of our samples were polished. Samples from Sumitomo and Element Six were also polished, until no trace of growth could be found on any surfaces. Comparing the three samples (Figure $5)$, the yellow color of our cubic crystal is more uniform, and no obvious color differences found across any surfaces. Minor differences in the yellow color were present in the sample from Sumitomo Corp., with the yellow color being much heavier in central region. This could be the result of different growth rates about the seed crystal. However, more substantial color differences are present in different growth sectors of the diamond sample from Elements Six Corp. The yellow color is not uniform, even in the same growth sector, and the color of the center region is heavier than in other regions. Further, $\{110\}$ growth sectors are clearly present in the sample, as shown in Figure 5(c). As the nitrogen content in a $\{110\}$ growth sector is much lower than that of a $\{100\}$ or $\{111\}$ face, the $\{110\}$ faces are almost colorless [12].

Micro-Raman spectroscopy measurements on the three diamond samples were carried out in different regions of the $\{100\}$ faces, and the full width at half maximum (FWHM) of the $1332 \mathrm{~cm}^{-1}$ vibration was used to characterize the degree of crystal lattice distortion or crystalline quality of large diamond crystals as detailed by Sumitomo Corp. [15].

The Raman peak data and FWHM of the three diamond samples are presented in Table 2. The diamond Raman peaks of the three samples are all near $1332 \mathrm{~cm}^{-1}$. The FWHW of the Sumitomo sample is the smallest, and the Element Six sample is the largest. In view of market prices, the prices of the Sumitomo diamond crystals are much higher than the Element Six diamonds, and this may be because they have better crystallinity.

The Element Six sample and our diamond sample also exhibit a small peak in the $1400-1450 \mathrm{~cm}^{-1}$, as shown in Figure 6, while this is not seen in the Sumitomo sample. This feature may be related to the inclusion of different impurities in the diamond lattice. Since the metal solvent in Sumitomo Corp. is reported to be a Fe-Co alloy [12], and, like us, Element Six use a Fe-Ni alloy, this peak may be related to the type of impurity being trapped in the diamond lattice from the metal solvents.

The nitrogen content of diamond can also be determined by IR absorption spectroscopy in the one-phonon zone [16]. The three samples were measured by micro-zone IR characterization on an FTIR. According to the calculation [16], the nitrogen content of the Sumitomo sample was $150 \mathrm{ppm}$, of the Element Six sample was 113 ppm, while the content of our samples was the largest, at $240 \mathrm{ppm}$.

The nitrogen contamination is related to the metal solvent. Sumitomo Corp., uses a Fe-Co alloy [12], while Element Six Corp. has not reported their solvent but it is assumed to be an Fe-Ni alloy. The new Fe-Ni alloy used in our lab is most likely the cause of the increase in nitrogen content in our diamond.

Again, the nitrogen content of diamond is closely related to the growth rate, with nitrogen content increase with increased growth rate [17]. As our samples have the highest

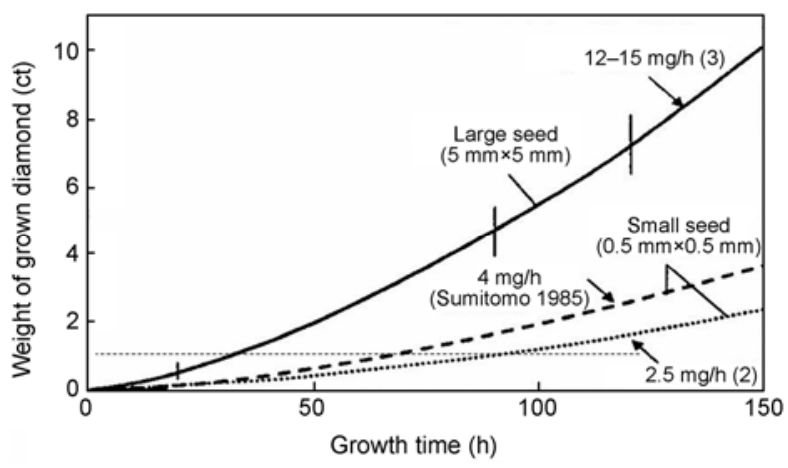

Figure 4 Growth rates of Ib diamond crystals at Sumitomo Corp.
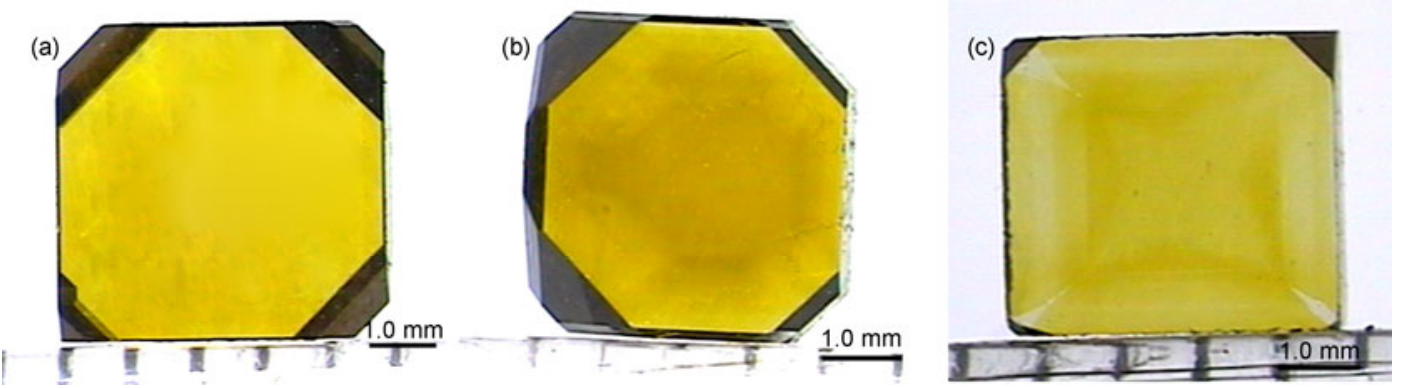

Figure 5 (Color online) Color uniformity of diamond crystal samples. (a) Our lab; (b) Sumitomo Corp.; (c) Element Six Corp. 
Table 2 Raman peak and FWHW of different diamond samples

\begin{tabular}{lcc}
\hline & Diamond peak $\left(\mathrm{cm}^{-1}\right)$ & FWHW $\left(\mathrm{cm}^{-1}\right)$ \\
\hline Sumitomo & 1333.10 & 5.298 \\
Element Six & 1332.98 & 5.837 \\
Our lab & 1332.88 & 5.570 \\
\hline
\end{tabular}
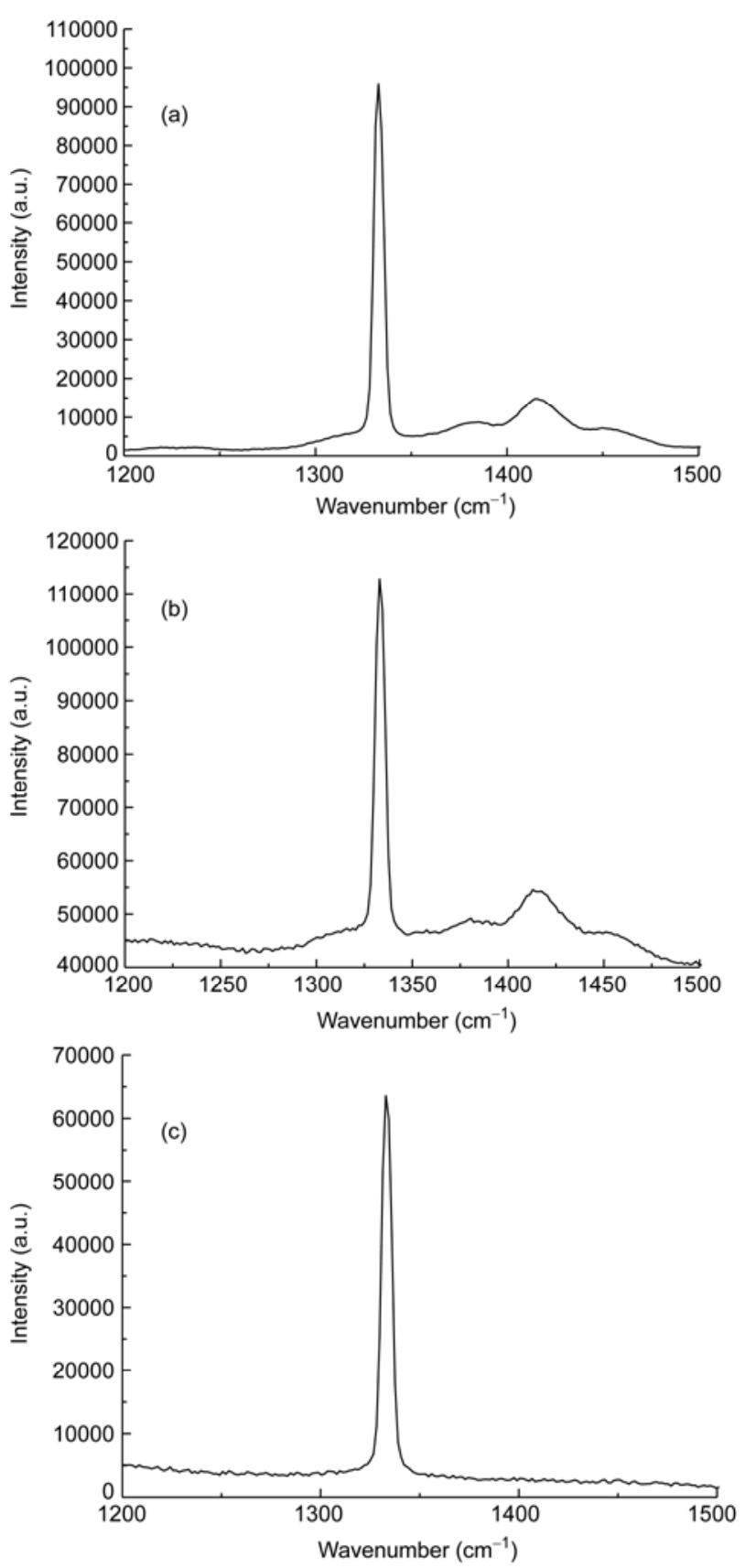

Figure 6 Raman spectrum of cubic diamond samples. (a) Our lab; (b) Element Six; (c) Sumitomo Corp.

growth rate, it follows that our nitrogen content should be the largest, although the growth rate of the Element Six sample has not been reported in recent references. Higher
Table 3 Nitrogen content of different diamond samples

\begin{tabular}{lcccc}
\hline & Face & $\begin{array}{c}\text { Point A } \\
(\mathrm{ppm})\end{array}$ & $\begin{array}{c}\text { Point B } \\
(\mathrm{ppm})\end{array}$ & $\begin{array}{c}\text { Average value } \\
(\mathrm{ppm})\end{array}$ \\
\hline Sumitomo Corp. & $(100)$ & 161 & 139 & 150 \\
Element Six Corp. & $(100)$ & 117 & 108 & 113 \\
Our lab & $(100)$ & 233 & 247 & 240 \\
\hline
\end{tabular}

growth rates also affect the crystal quality, resulting in more crystal defects. Therefore, the crystalline quality of our samples may be lower than that of Sumitomo samples, and this also agrees with the Raman spectroscopy results.

In summary, using the HPHT temperature gradient method at $5.4 \mathrm{GPa}$ and $1500 \mathrm{~K}$, with a new Fe-Ni alloy as solvent metal, large high-quality cubic diamond crystals have been grown at high growth rates.

(1) The highest growth rate achieved was $7.3 \mathrm{mg} / \mathrm{h}$. Over the same time period, about $30 \mathrm{~h}$, this growth rate is higher than the large seed method of Sumitomo Corp. $(6.7 \mathrm{mg} / \mathrm{h})$, despite the use in our lab of a $0.8 \mathrm{~mm}$ seed crystal.

(2) The radical growth rates (horizontal direction) of the large cubic diamond crystals were higher than the axial growth rates (vertical direction). The crystal was $7.3 \mathrm{~mm}$ across, while the weight was only 1.2 ct.

(3) Compared with high quality cubic diamond crystal samples made by Sumitomo and Element Six Corp., the yellow color of our samples was more uniform. No other growth sectors were present, while in the Element Six sample, $\{110\}$ growth sectors were found. The Raman FWHW of the $1332 \mathrm{~cm}^{-1}$ peak in our diamond sample was smaller than that of Element six, but larger than that of Sumitomo. The nitrogen content of our diamond samples was $240 \mathrm{ppm}$, which was higher than that of the Sumitomo and Element Six samples, and this was found to be related to the higher growth rates of our diamond samples.

This work was supported by the Natural Science Foundation of the Education Department of Henan Province (2009A430014).

1 Yarnell A. The many facets of man-made diamonds. Cenear, 2004, 82: 26-31

2 Kanda H, Ohsawa T. Effect of solvent metals upon the morphology of synthetic diamonds. J Crystal Growth, 1989, 94: 115-124

3 Strong H M, Wentorf R H Jr. The growth of large diamond crystals. Naturwissenschaften, 1972, 59: 1-7

4 Sumiya H, Toda N, Satoh S. High-pressure synthesis of high-purity diamond crystal. Diamond Relat Mater, 1996, 5: 1359-1365

5 Bundy F P, Strong H M, Wentorf R H Jr. Methods and mechanisms of synthetic diamond growth. Chem Phys Carbon, 1973, 10: 213-263

6 Burns R C, Hansen J O, Spits R A, et al. Growth of high purity large synthetic diamond crystals. Diamond Relat Mater, 1999, 8: 14331437

7 Xiao H Y, Jia X P, Ma H A, et al. Synthesis of high quality type-Ib diamond crystals in carats grade. Chin Sci Bull, 2010, 55: 1372-1375

8 Ma L Q, Ma H A, Xiao H Y, et al. Effect of additive boron on type-Ib gem diamond single crystals synthesized under HPHT. Chin Sci Bull, 2010, 55: 677-679 
9 Yan C S, Mao H K, Li W, et al. Ultrahard diamond single crystals from chemical vapor deposition. Phys Status Solidi A, 2004, 201: R24-R27

10 Liang Q, Yan C S, Meng Y F, et al. Recent advances in high-growth rate single-crystal CVD diamond. Diamond Relat Mater, 2009, 18: 698-703

11 Sumiya H, Toda N, Satoh S. High quality large diamond crystals. New Diamond Front Carbon Tech, 2000, 10: 233-251

12 Sumiya H, Toda N, Satoh S. Growth rate of high-quality large diamond crystals. J Crystal Growth, 2002, 237-239: 1281-1285

13 Strong H M, Hanneman R E. Crystallization of diamond and graphite. J Chem Phys, 1967, 46: 3668-3673
14 Burns R C, Cvetkovic V, Dodge C N, et al. Growth-sector dependence of optical features in large synthetic diamonds. J Cryst Growth, 1990, 140: 257-279

15 Sumiya H, Toda N, Nishibayashi Y, et al. Crystalline perfection of high purity synthetic diamond crystal. J Crystal Growth, 1997, 178: 485-490

16 Zhang Y F, Zang C Y, Ma H A, et al. HPHT synthesis of large single crystal diamond doped with high nitrogen concentration. Diamond Relat Mater, 2008, 17: 209-211

17 Kiflawi I, Kanda H, Lawson S C. The effect of the growth rate on the concentration of nitrogen and transition metal impurities in HPHT synthetic diamonds. Diamond Relat Mater, 2002, 11: 204-211

Open Access This article is distributed under the terms of the Creative Commons Attribution License which permits any use, distribution, and reproduction in any medium, provided the original author(s) and source are credited. 